\title{
Quantitative, Real-Space Statistical Analysis of Imperfect Lattices
}

Benjamin H. Savitzky ${ }^{1}$, Robert Hovden ${ }^{2}$, Kevin Whitham ${ }^{3}$, Tobias Hanrath ${ }^{4}$ and Lena F. Kourkoutis ${ }^{2,5}$

1. Dept. of Physics, Cornell University, Ithaca, NY 14853, USA.

2. School of Applied \& Engineering Physics, Cornell University, Ithaca, NY 14853, USA.

3. Dept. of Materials Science \& Engineering, Cornell University, Ithaca, NY 14853, USA.

4. School of Chemical \& Biomolecular Engineering, Cornell University, Ithaca, NY 14853, USA.

5. Kavli Institute for Nanoscale Science, Cornell University, Ithaca, NY 14853, USA.

Rapid advances in both instrumentation and analysis have enabled aberration corrected STEM position measurements with precision far surpassing atomic length scales [1]. Local distortions around defects or interfaces can, therefore, be readily studied. However correlated structural deformations at longer length scales can also have dramatic effects on material properties, such as in the case of propagating disorder. Here, we describe a method of analysis which strikes a balance between the need for local as well as medium-range statistical information based on the two-dimensional vectorial pair correlation function, which provides an interpretable, statistical picture of local structure in real space.

As an illustrative example, we examine PbSe quantum dots (QDs) which have been epitaxially connected across the $\{100\}$ facets, and form square superlattices (SLs) over several micron grains (Fig. 1a). Theoretical work on these systems predicts highly tunable band structures exhibiting novel physics, however, charge carrier delocalization is currently limited by disorder to a few QD spacings [2], implying the need for detailed analysis of disorder in the QD SL. Using sub-pixel QD centroid positions extracted from STEM micrographs, we calculated the two dimensional pair correlation function $G(\boldsymbol{r})=\int n\left(\boldsymbol{r}^{\prime}\right) n\left(\boldsymbol{r}-\boldsymbol{r}^{\prime}\right) d \boldsymbol{r}^{\prime}$ for particle density $n(\mathbf{r})$, Fig. 1b. $G(\boldsymbol{r})$ describes the likelihood of finding a particle at position $\mathbf{r}$ given a particle at the origin. The anisotropy of the peaks indicates greater shearing disorder than compressive/tensile disorder, while the broadening of peaks progressively farther from the origin indicates propagation of translational disorder through the SL, which we describe numerically using the standard deviation $\sigma$ of Gaussian fits to peaks in $G(\boldsymbol{r}) n$ lattice spacings from the origin (Fig 1c). The growth of $\sigma$ with $n$ is shown to agree with a real paracrystal model, which incorporates both propagating and non-propagating disorder terms (Fig 1d,e). If the subset of QD pairs that are not epitaxially connected is excluded from this analysis, nearest-neighbor order is shown to improve dramatically, implying that connectivity has a disproportionately large effect at long ranges due to propagation of disorder.

In Fig. 2, we show that SL order improves in thicker samples by plotting the values of $\sigma$ (longitudinal and transverse to $\boldsymbol{r}$ ) for adjacent QDs over samples of varying thicknesses. Due to the projection nature of STEM imaging, the $G(\mathbf{r})$ maps for thicker samples describe the relationship between average positions of QD columns; to account for this, we calculated the $\sigma$ values for single 2D layers of the SL structure using the models and parameters extracted as described in Fig. 1, enabling comparison between grains of different thicknesses.

Local analysis methods such as real space particle-particle distance mapping may overlook a simple statistical description governing lattice structure such as the paracrystal model. Fourier space methods such as diffraction or FFT analysis sacrifice the ability to relate local features in real space such as connectivity to an appropriate statistical description. While this QD system provides an exemplar of the method, we also demonstrate the benefit of this analysis when applied to atomic lattice structures. 
[1] X. Sang, J.M. LeBeau, Ultramicroscopy 138 (2014), 28-35

[2] K. Whitham, J. Yang, B.H. Savitzky, L.F. Kourkoutis, F. Wise, T. Hanrath, Nature Materials (2016), (in press)

[3] BHS acknowledges support from the NSF GRFP (DGE-1144153). This work was supported by the Cornell Center for Materials Research with funding from the NSF MRSEC program (DMR-1120296)
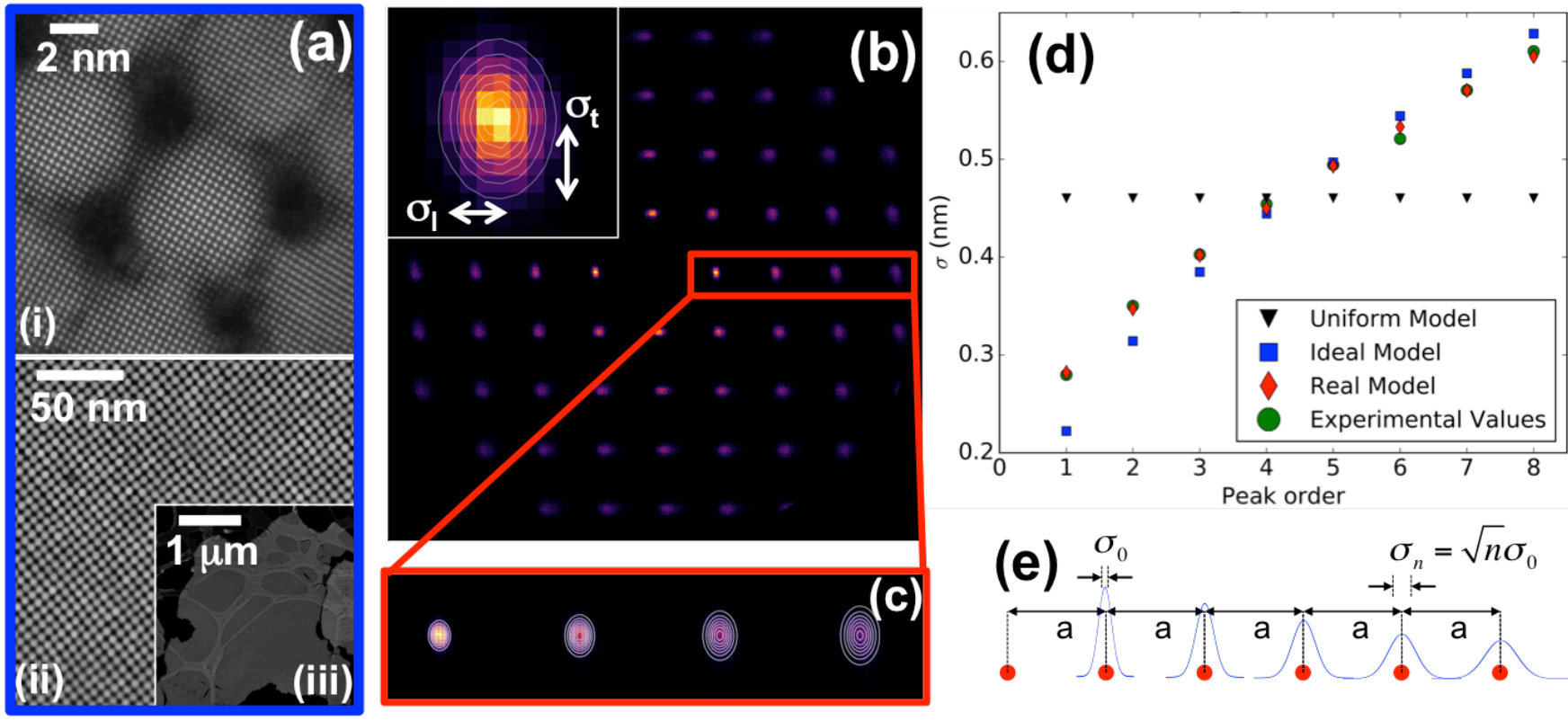

Figure 1. The 2D vectorial pair correlation function $G(\boldsymbol{r})$ reveals the statistical behavior governing propagation of disorder in epitaxially connected $\mathrm{PbSe}$ quantum dot superlattices. Subpixel centroid positions from aberration corrected STEM micrographs (a) are used to calculate $G(\boldsymbol{r})(\mathrm{b})$. Broadening peaks in both the longitudinal and transverse directions (b, inset) progressively farther from the origin indicate propagation of disorder through the superlattice (c). The rate of broadening is compared to several models of crystalline disorder, including a uniform model, the ideal paracrystal model (e), and the real paracrystal model (d). The system is well described by a real paracrystal model.

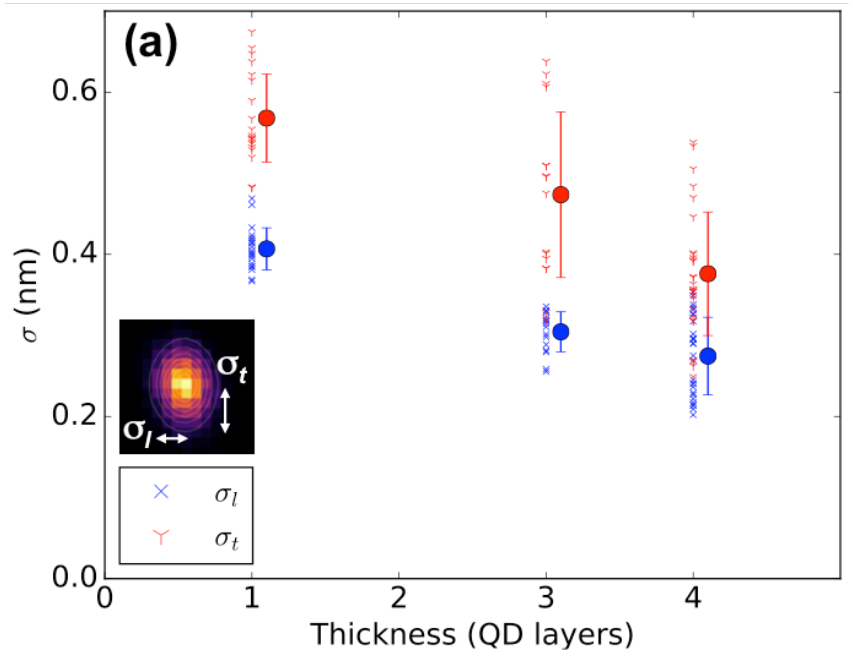

Figure 2. Statistical analysis of multiple superlattices demonstrating improved order with increasing sample thickness. The standard deviation in the nearestneighbor probability distribution, determined from $G(\boldsymbol{r})$ over many datasets, decreases as sample thickness is increased. This data has been corrected for projection using the real paracrystalline parameters extracted following the method described in Fig. 1. 